\title{
Effects of exercise training on sleep apnoea in patients with coronary artery disease: a randomised trial
}

\author{
Monique Mendelson ${ }^{1,2}$, Owen D. Lyons ${ }^{1,2,3}$, Azadeh Yadollahi ${ }^{1,2,4}$, \\ Toru Inami ${ }^{1,2,3}$, Paul $\mathrm{Oh}^{5}$ and T. Douglas Bradley ${ }^{1,2,3}$
}

Affiliations: ${ }^{1}$ Sleep Research Laboratory, University Health Network Toronto Rehabilitation Institute, Toronto, ON, Canada. ${ }^{2}$ Centre for Sleep Medicine and Circadian Biology, University of Toronto, Toronto, ON, Canada. ${ }^{3}$ Dept of Medicine, University Health Network, Toronto General Hospital, Toronto, ON, Canada. ${ }^{4}$ Institute of Biomaterial and Biomedical Engineering, University of Toronto, Toronto, ON, Canada. ${ }^{5}$ Cardiac Rehabilitation and Prevention, University Health Network Toronto Rehabilitation Institute, Toronto, ON, Canada.

Correspondence: T. Douglas Bradley, University Health Network Toronto General Hospital, 9N-943, 200 Elizabeth Street, Toronto, ON, M5G 2C4, Canada. E-mail: douglas.bradley@utoronto.ca

ABSTRACT Overnight fluid shift from the legs to the neck and lungs may contribute to the pathogenesis of obstructive sleep apnoea (OSA) and central sleep apnoea (CSA). We hypothesised that exercise training will decrease the severity of OSA and CSA in patients with coronary artery disease (CAD) by decreasing daytime leg fluid accumulation and overnight rostral fluid shift.

Patients with CAD and OSA or CSA (apnoea-hypopnoea index $>15$ events per $\mathrm{h}$ ) were randomised to 4 weeks of aerobic exercise training or to a control group. Polysomnography, with measurement of leg, thoracic and neck fluid volumes and upper-airway cross-sectional area (UA-XSA) before and after sleep, was performed at baseline and follow-up.

17 patients per group completed the study. Apnoea-hypopnoea index decreased significantly more in the exercise group than in the control group $(31.1 \pm 12.9$ to $20.5 \pm 9.4$ versus $28.1 \pm 13.5$ to $27.0 \pm 15.1$ events per $h, p=0.047)$, in association with a greater reduction in the overnight change in leg fluid volume $(579 \pm 222$ to $466 \pm 163$ versus $453 \pm 164$ to $434 \pm 141 \mathrm{~mL}, \mathrm{p}=0.04)$ and by a significantly greater increase in the overnight change in UA-XSA in the exercise group $(\mathrm{p}=0.04)$.

In patients with CAD and sleep apnoea, exercise training decreases sleep apnoea severity via attenuation of overnight fluid shift and an increase in UA-XSA.

@ERSpublications

In patients with $\mathrm{CAD}$ and moderate-to-severe sleep apnoea, exercise training reduces AHI during sleep http://ow.ly/YvQzS

Editorial comment in: Eur Respir J 2016; 48: 23-25.

This article has supplementary material available from erj.ersjournals.com

Received: Nov 142015 | Accepted after revision: Feb 12 2016 | First published online: April 132016

Clinical trial: This study is registered at www.controlled-trials.com with identifier number ISRCTN50108373

Support statement: This study was supported by a Canadian Institutes of Health Research operating grant MOP-82731. O.D. Lyons is supported by a joint Canadian Thoracic Society/European Respiratory Society Peter Macklem Research Fellowship and the Joseph M. West Family Memorial Fund Postgraduate Research Award. T. Inami was supported by an unrestricted fellowship from Philips Respironics Japan. T.D. Bradley is supported by the Clifford Nordal Chair in Sleep Apnoea and Rehabilitation Research. Funding information for this article has been deposited with FundRef.

Conflict of interest: None declared.

Copyright OERS 2016 


\section{Introduction}

Among patients with coronary artery disease (CAD), those who participate in a cardiac rehabilitation exercise programme have a significantly lower mortality rate than those who do not [1]. The mechanisms underlying such exercise-induced improvements in mortality have yet to be elucidated but probably involve beneficial effects on endothelial and platelet function, and on inflammatory markers [1, 2]. In patients with CAD, untreated obstructive sleep apnoea (OSA) is associated with an increased risk of cardiovascular morbidity and mortality [3]. Untreated OSA is also associated with increased cardiovascular mortality in the general population [4], and both OSA and central sleep apnoea (CSA) are associated with increased mortality in patients with heart failure $[5,6]$. Exercise training has been shown to reduce the severity of OSA and CSA in patients with and without heart failure [7-9]. Thus, an additional beneficial effect of exercise in CAD patients may be attenuation of sleep apnoea.

OSA is characterised by complete or partial upper-airway collapse during sleep [10] while CSA is caused by complete or partial reductions in central neural outflow to the respiratory muscles during sleep [11]. Both CSA and OSA have adverse effects on the cardiovascular system by causing intermittent hypoxia, increased sympathetic nervous system activation and vascular endothelial dysfunction [10, 11].

It has been shown that fluid accumulates in the legs while one is seated during the day, due to gravity and lack of activity of the musculovenous pump of the calves, and upon lying down at night, this fluid is redistributed rostrally by gravity [12]. Nocturnal rostral fluid shift can play a role in the pathogenesis of both OSA and CSA, with the type of sleep apnoea being related to whether fluid shift from the legs is redistributed predominantly to the neck or chest, respectively $[13,14]$. Fluid redistribution into the neck may lead to increased tissue pressure surrounding the upper airway, reducing its size and increasing its collapsibility, thus predisposing to OSA [12]. Fluid redistribution into the chest may stimulate pulmonary irritant receptors provoking hyperventilation and hypocapnia, driving carbon dioxide tension below the apnoeic threshold, predisposing to CSA $[13,14]$. Interventions that reduce fluid accumulation in the legs and nocturnal rostral fluid shift, such as diuretics, compression stockings, ultrafiltration and physical activity, can attenuate both OSA and CSA [15-18].

The aim of this study was to test the hypothesis that exercise training, by engaging the musculovenous pump in the calves and reducing fluid retention in the legs, will lead to a reduction in nocturnal rostral fluid shift and a decrease in the apnoea-hypopnoea index (AHI) in patients with CAD and sleep apnoea.

\section{Methods}

Study design

This was a 4-week, single-centre, unblinded, parallel-group randomised controlled trial.

\section{Subjects}

Inclusion criteria were subjects aged 18-85 years with CAD, defined as a documented myocardial infarction, coronary artery bypass surgery or coronary angioplasty, and/or stenting, referred to the University Health Network Toronto Rehabilitation Institute (Toronto, ON, Canada) for cardiac rehabilitation, with a sleep apnoea disorder defined as at least 15 apnoeas and hypopnoeas per hour of sleep (AHI). Exclusion criteria were treated OSA or CSA, adenotonsillar hypertrophy, use of diuretics and inability to exercise due to musculoskeletal problems.

\section{Cardiopulmonary exercise test}

Subjects underwent symptom-limited, graded exercise tests on a cycle ergometer (800 P; Ergoline, Bitz, Germany) or a treadmill (Quinton, Bothwell, WA, USA) (see online supplemental material for details).

\section{In-centre exercise training}

Subjects in the exercise group participated in an aerobic exercise training programme, which included $30 \mathrm{~min}$ of moderate-intensity walking per day, 5 days per week. The walking prescription was set at a distance of approximately $1.6-2.0 \mathrm{~km}$ and an intensity equivalent to $60 \%$ of peak oxygen consumption $\left(V^{\prime} \mathrm{O}_{2}\right.$ peak), assessed via the heart rate response at $60 \% V^{\prime} \mathrm{O}_{2}$ peak during the maximum exercise test. Each exercise session began with a 10-min warm-up followed by $30 \mathrm{~min}$ of moderate-intensity walking on a track or treadmill. The exercise session was concluded with a 10-min cool-down. Participants attended the Cardiac Rehabilitation Centre for supervised exercise training three times per week and performed their exercise prescription at home the other 2 days. 
(see online supplementary material for details). The participants in the control group did not wear the pedometer, in order to ensure that this device was not providing feedback that might encourage them to increase their activity levels. They did, however, complete the short version of the International Physical Activity Questionnaire (IPAQ) to ensure that they did not increase their activity levels during the control period. For the IPAQ, metabolic equivalent task (MET) minutes were computed by multiplying pre-defined MET scores by the minutes of a specific activity performed to weigh each type of activity by its energy requirement (i.e. for walking, 3.3 was used as the MET score). Exercise compliance for patients in the intervention group was calculated as the total number of exercise sessions completed over the 4 -week period reported in the exercise training diaries, and expressed as a percentage of the total number of sessions and as a percentage of total prescribed minutes. Home-based exercise compliance was verified by examining exercise training diaries and pedometer values for the 2 days of the week that patients were not on site. These data were verified at every on-site visit and transferred into a compliance chart.

\section{Polysomnography}

All subjects underwent overnight polysomnography (PSG) using standard techniques and scoring criteria for sleep stages, arousals from sleep and periodic leg movements (PLMs) [19, 20] (see online supplementary material for details). Apnoeas were defined as $\geqslant 90 \%$ reduction in airflow or thoracoabdominal motion from baseline, respectively, lasting $\geqslant 10 \mathrm{~s}$. Hypopnoeas were defined as $\geqslant 30 \%$ reduction airflow lasting $\geqslant 10 \mathrm{~s}$, associated with a $\geqslant 3 \%$ desaturation or an arousal from sleep [8]. They were classified as obstructive if there was out-of-phase thoracoabdominal motion or flow limitation on the nasal pressure tracing, and central if there was an absence of thoracoabdominal motion, or in-phase thoracoabdominal motion without evidence of airflow limitation, during apnoeas and hypopnoeas, respectively [20]. Sleep apnoea was defined as an AHI $\geqslant 15$ events per $\mathrm{h}$ and was classified as OSA when $\geqslant 50 \%$ of events were obstructive and as CSA when $>50 \%$ of events were central. The AHI and PLM indices were calculated. Signals were recorded on a computerised sleep recording system (Sandman; Nellcor Puritan Bennett Ltd., Ottawa, ON, Canada), and scored by technicians blind to the other experimental data and to the randomisation.

\section{Fluid volumes, and neck and calf circumferences}

Weight was measured before going to bed and within $30 \mathrm{~min}$ of awakening the next morning, before urinating. With subjects instrumented for PSG, lying awake and supine, right and left leg fluid volume (LFV), thoracic fluid volume (TFV), and neck fluid volume (NFV) were measured simultaneously using a bioelectrical impedance spectrum analyser (MP150; Biopac Systems Canada, Inc., Montreal, QC, Canada), as previously described [16]. This well-validated technique uses impedance to electrical current within a body segment to measure its fluid content [21]. Neck circumference and calf circumferences were measured by tape measure as previously described [12]. Measurements of LFV, TFV, NFV, and neck and calf circumferences were repeated the next morning after awakening, before subjects got out of bed, and the overnight changes calculated. Measurements were made before PSGs were scored by the experimenter, who was blind to the PSG findings and AHI. Similarly, PSGs were scored by personnel blind to fluid volume, and calf and neck circumference measurements.

Upper-airway cross-sectional area

Intraluminal upper-airway cross-sectional area (UA-XSA) before and after sleep was measured by acoustic pharyngometry (Eccovision; Hood Laboratories, Pembroke, MA, USA), with the patient lying supine and the head in the neutral position as previously described [22].

\section{Protocol}

Prior to entering cardiac rehabilitation, subjects underwent a baseline cardiopulmonary exercise test to assess their suitability for cardiac rehabilitation. They were then approached to participate in our study prior to commencing cardiac rehabilitation. Those who agreed provided written informed consent prior to participation. Participants then underwent a 1-week baseline pedometer step-taking assessment and a baseline PSG during which calf and neck circumferences, fluid volumes, and UA-XSA were assessed as described above. Individuals with an AHI $\geqslant 15$ events per $\mathrm{h}$ were then randomly allocated to an exercise intervention or control group according to a computer-generated random schedule in permuted blocks of two and four. Participants in the control group were instructed to maintain their usual level of activity levels for 4 weeks. None of the subjects was treated for their sleep apnoea during the study period. After 4 weeks, all assessments made at baseline were repeated. The study protocol was approved by the Research Ethics Board of the University Health Network (Toronto, ON, Canada). 


\section{Data analysis}

Continuous variables are presented as mean \pm SD and categorical variables as proportions. Results were analysed on an intention-to-treat basis irrespective of exercise adherence. Baseline data were compared between groups using unpaired t-tests, Mann-Whitney U-tests or Chi-squared tests. Changes from baseline to follow-up within each group were analysed using paired t-tests or Wilcoxon tests as appropriate. Repeated-measures ANOVA was used to assess changes in variables from baseline to follow-up between groups. For the AHI, we performed an ANCOVA, to adjust for any change in supine time from baseline to follow-up. A p-value of $<0.05$ was considered significant. A sample size was calculated assuming a 35\% reduction in AHI and a standard deviation of $35 \%$ in the intervention group based on previous studies [7-9, 23], and a two-tailed $\alpha$ of 0.05 and $\beta$ of $80 \%$. The resulting sample size was 18 patients per group. Assuming attrition of four subjects per group, we estimated 22 subjects per group. Statistical analyses were performed using SPSS 20 (SPSS Inc., Chicago, IL, USA).

\section{Results}

\section{Subjects}

Study subjects' flow through the protocol is shown in figure 1.62 subjects meeting eligibility criteria who had undergone a baseline cardiopulmonary exercise test underwent PSG between September 2014 and April 2015. 18 patients had an AHI $<15$ events per $\mathrm{h}$ and were not randomised. The remaining 44 had an $\mathrm{AHI} \geqslant 15$ events per $\mathrm{h}$ and were randomised. Of these, five patients discontinued before study completion in both the exercise and control groups, such that 17 subjects per group completed the protocol. Reasons for study withdrawal in the exercise group were health-related for two patients (gout and worsening of diabetic retinopathy) and another three patients were lost to follow-up before study completion (figure 1). In the control group, one patient changed their medication during the study period (began a diuretic treatment) and the other four patients were lost to follow-up. Characteristics of the subjects who completed the protocol are shown in table 1.

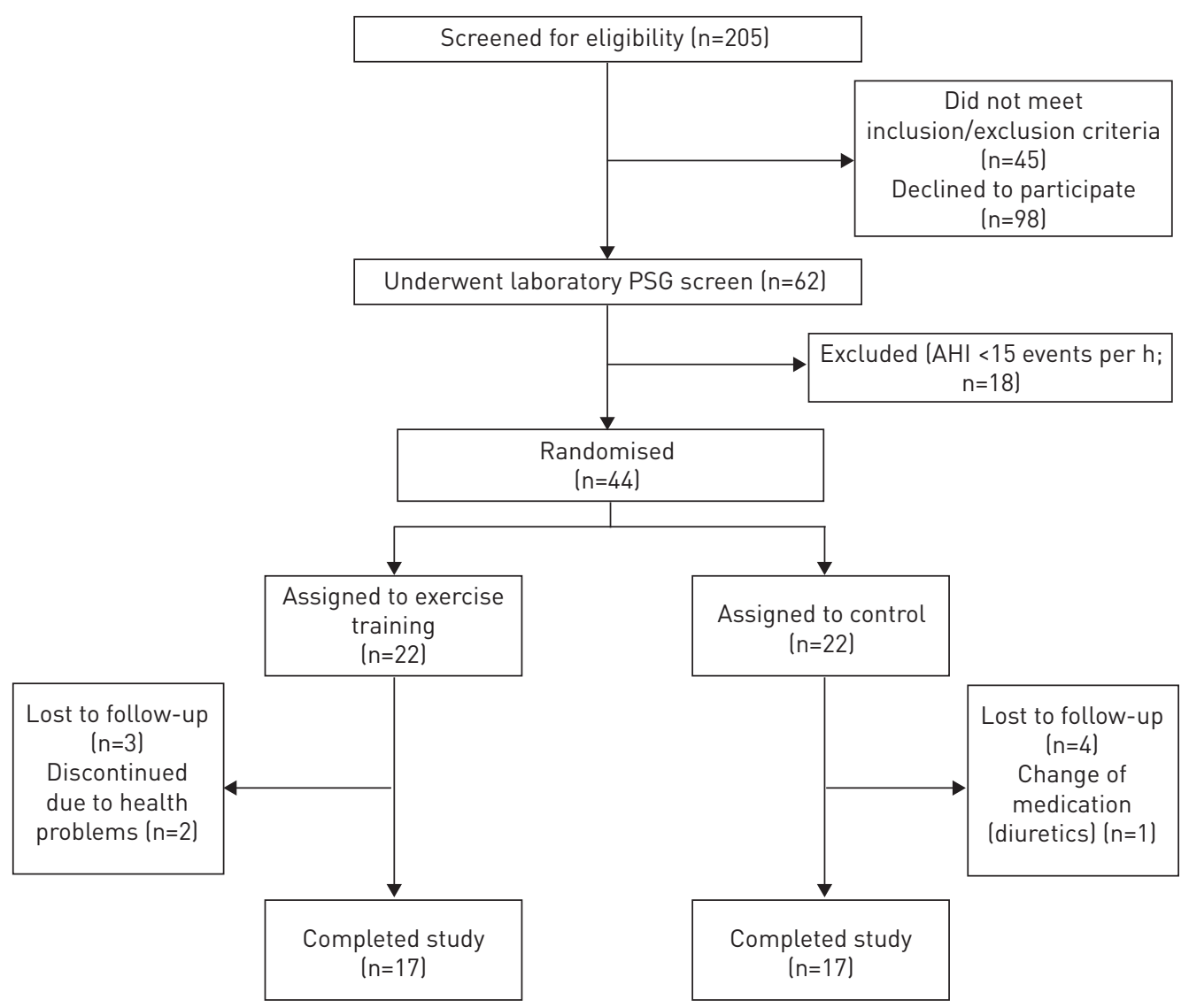

FIGURE 1 Study participant flow chart. PSG: polysomnography; AHI: apnoea-hypopnoea index. 
TABLE 1 Baseline characteristics of the subjects

\begin{tabular}{lccc} 
& Exercise & Control & p-value \\
\hline Subjects $\mathbf{n}$ & 17 & 17 & \\
Age years & $63.8 \pm 8.0$ & $59.6 \pm 11.8$ & 0.24 \\
Male & $16(94)$ & $14(82)$ & 0.51 \\
BMI kg.m ${ }^{-2}$ & $28.6 \pm 4.5$ & $26.2 \pm 3.9$ & 0.11 \\
Cardiovascular history & $11(65)$ & & \\
MI & $11(65)$ & $13(76)$ & 0.61 \\
PCl & $6(35)$ & $13(76)$ & 0.34 \\
CABG & & $3(18)$ & 0.34 \\
Comorbidities & $6(35)$ & & \\
Type 2 diabetes & $13(76)$ & $4(24)$ & 0.52 \\
Hypertension & $3(18)$ & $13(76)$ & 1.00 \\
Atrial fibrillation & $14(82)$ & $1(6)$ & 0.35 \\
Medication & $4(24)$ & $15(88)$ & 0.89 \\
$\beta-b l o c k e r$ & $17(100)$ & $2(12)$ & 0.51 \\
Calcium channel blocker & $6(35)$ & $16(94)$ & 0.34 \\
Antiplatelet agent & $3(18)$ & $9(53)$ & 0.49 \\
ACE inhibitor & $16(94)$ & $4(24)$ & 0.46 \\
ARBs & $3913.3 \pm 1118.9$ & $15(88)$ & 0.63 \\
Lipid-lowering agent & $31.1 \pm 12.9$ & $4061.3 \pm 1243.5$ & 0.91 \\
Physical activity level steps per day & $15 / 2$ & $28.1 \pm 13.5$ & 0.53 \\
AHI events per $\mathbf{h}$ & & $13 / 4$ & 0.66 \\
OSA/CSA & & \\
\hline
\end{tabular}

Data are presented as $\mathrm{n}$, mean \pm SD or $\mathrm{n}(\%)$, unless otherwise stated. BMI: body mass index; MI: myocardial infarction; $\mathrm{PCl}$ : percutaneous coronary intervention; CABG: coronary artery bypass graft; ACE: angiotensin-converting enzyme; ARB: angiotensin II receptor blocker; AHI: apnoea-hypopnoea index; OSA: obstructive sleep apnoea; CSA: central sleep apnoea.

\section{Exercise data}

There were no significant between-group differences in any of the cardiopulmonary variables from baseline to follow-up (table 2). Participants in the exercise group increased their daily physical activity levels significantly from baseline to week 4 (i.e. from $3913.3 \pm 1100.5$ to $8228.5 \pm 1125.9$ steps per day, $\mathrm{p}<0.0001)$. Participants in the exercise group completed a mean \pm SD of $87.5 \pm 7.7 \%$ of their exercise sessions. This corresponded to completing $92.5 \pm 8.1 \%$ of the prescribed minutes of exercise. The control group did not increase their activity levels as measured by the walking component of the IPAQ (i.e. $388.5 \pm 267.4$ to 403.1 $\pm 289.1 \mathrm{MET} \cdot \mathrm{min}$ per week).

\section{PSG data}

At follow-up, the exercise group experienced a significant (34\%) decrease in the AHI, which was greater than in the control group ( $\mathrm{p}=0.047)$ (figure 2 and table 3 ). There was a significantly greater reduction in the obstructive AHI in the exercise group than in the control group $(p=0.04)$. At follow-up, the exercise

TABLE 2 Changes in cardiopulmonary exercise test variables

\begin{tabular}{|c|c|c|c|c|c|}
\hline & \multicolumn{2}{|c|}{ Exercise $^{\#}$} & \multicolumn{2}{|c|}{ Control $^{\#}$} & Between-group p-value \\
\hline$V^{\prime} \mathrm{O}_{2}$ peak $\mathrm{mL} \cdot \mathrm{kg}^{-1} \cdot \mathrm{min}^{-1}$ & $20.4 \pm 4.0$ & $21.8 \pm 4.1^{*}$ & $22.5 \pm 8.4$ & $23.1 \pm 8.3$ & 0.31 \\
\hline$V^{\prime} \mathrm{O}_{2}$ at $\mathrm{AT} \mathrm{mL} \cdot \mathrm{kg}^{-1} \cdot \mathrm{min}^{-1}$ & $17.5 \pm 5.6$ & $18.4 \pm 7.3$ & $18.4 \pm 7.3$ & $17.5 \pm 5.6$ & 0.74 \\
\hline Heart rate at AT beats per min & $104.8 \pm 15.9$ & $103.7 \pm 21.8$ & $103.7 \pm 21.8$ & $104.8 \pm 15.9$ & 0.48 \\
\hline Resting heart rate beats per min & $66.1 \pm 10.3$ & $64.3 \pm 12.3$ & $69.9 \pm 16.0$ & $69.4 \pm 14.5$ & 0.68 \\
\hline Peak heart rate beats per min & $116.6 \pm 17.6$ & $123.4 \pm 21.8^{*}$ & $126.3 \pm 24.8$ & $128.7 \pm 26.8$ & 0.21 \\
\hline
\end{tabular}

Data are presented as mean \pm SD unless otherwise stated. $V^{\prime} O_{2}$ peak: peak oxygen consumption; $V^{\prime} 0_{2}$ : oxygen consumption; AT: anaerobic threshold; RER: respiratory exchange ratio; RPE: rating of perceived exertion. ${ }^{\#}: n=15$, data unavailable in two patients. ${ }^{*}$ : $p<0.05$ between baseline and follow-up (within group). 
group experienced a significantly greater reduction in the non-rapid eye movement (REM) but not REM AHI ( $p=0.032$ and $p=0.25$, respectively) (figure 2 and table 3 ) than the control group. When the analysis was limited to men only, results were similar (online supplementary tables S1 and S2).

The small number of patients with CSA tended to respond in the same pattern as the patients with OSA. The two in the exercise group both experienced reductions in their AHI averaging 49\% (from a mean of 39.4 to 20.1 events per $\mathrm{h}$ ) whereas the four in the control group experienced a smaller reduction of $17 \%$ (from a mean of 34.6 to 28.8 events per h).

\section{Fluid volume changes and UA-XSA}

At follow-up, the exercise group experienced significantly greater reductions in evening LFV and in the overnight change in LFV than the control group ( $\mathrm{p}=0.042$ and $\mathrm{p}=0.023$, respectively) (table 4 ). Similarly, the exercise group experienced a greater reduction in the morning neck circumference and overnight change in neck circumference than the control group ( $\mathrm{p}=0.02$ and $\mathrm{p}=0.012$, respectively) (table 4). Morning UA-XSA and overnight change in UA-XSA increased significantly more in the exercise than the control group ( $\mathrm{p}=0.007$ and $\mathrm{p}=0.04$, respectively) (table 4$)$.

\section{Discussion}

Our study has given rise to several novel observations concerning the role of nocturnal fluid redistribution in the pathogenesis of OSA and CSA in patients with CAD. First, we demonstrated in a randomised controlled trial that 4 weeks of exercise training induced a significant reduction in the AHI in patients with CAD. Second, we showed that this reduction in AHI was accompanied by a reduction in the amount of fluid shifting out of the legs overnight. Third, reductions in the AHI and overnight fluid shift from the legs were accompanied by increases in UA-XSA and decreases in neck circumference assessed in the morning. These findings suggest that a reduction in overnight fluid shift from the legs to the neck with a consequent dilatation of the upper airway was a mechanism involved in the reduction in AHI in those with OSA, in the absence of any changes in physical fitness or body weight. In addition, the small number of patients with CSA tended to respond to exercise in the same pattern as the patients with OSA.

This is the first randomised trial to investigate the effects of exercise training on sleep apnoea in patients with CAD. Its findings extend those of four earlier studies on the effects of exercise training on sleep apnoea severity. In the first, a randomised controlled trial of 43 patients with OSA without comorbidities, 3 months of exercise training caused a $24 \%$ reduction in the AHI [7]. In the second, a nonrandomised uncontrolled study involving 18 patients with chronic heart failure and sleep apnoea, 6 months of aerobic exercise training was accompanied by a $64 \%$ decrease in the AHI, due mainly to a reduction in the central AHI [10]. The third was a nonrandomised cross-over study in which 25 patients with heart failure were stratified into three groups: OSA, CSA or no sleep apnoea [9]. After 4 months of exercise training, there was a $36 \%$ decrease in the AHI in the OSA group, similar to our findings, but no significant decrease in the CSA group. We had too few patients with CSA to do statistical comparisons, but as described above the two patients with CSA in the exercise group had a greater fall in their AHI than the four with CSA in the control group. Lastly, REDOLFI et al. [17] conducted a randomised, crossover study and showed that 1 week of walking twice daily for 45 min caused a 30\% reduction in the AHI in eight patients with OSA but without comorbidities, which occurred concomitantly with a reduction in the amount of fluid shifting from the legs to the neck overnight. The present study is generally consistent with those results but differed from that study in several important ways. First, it included a much larger sample size. Second, it
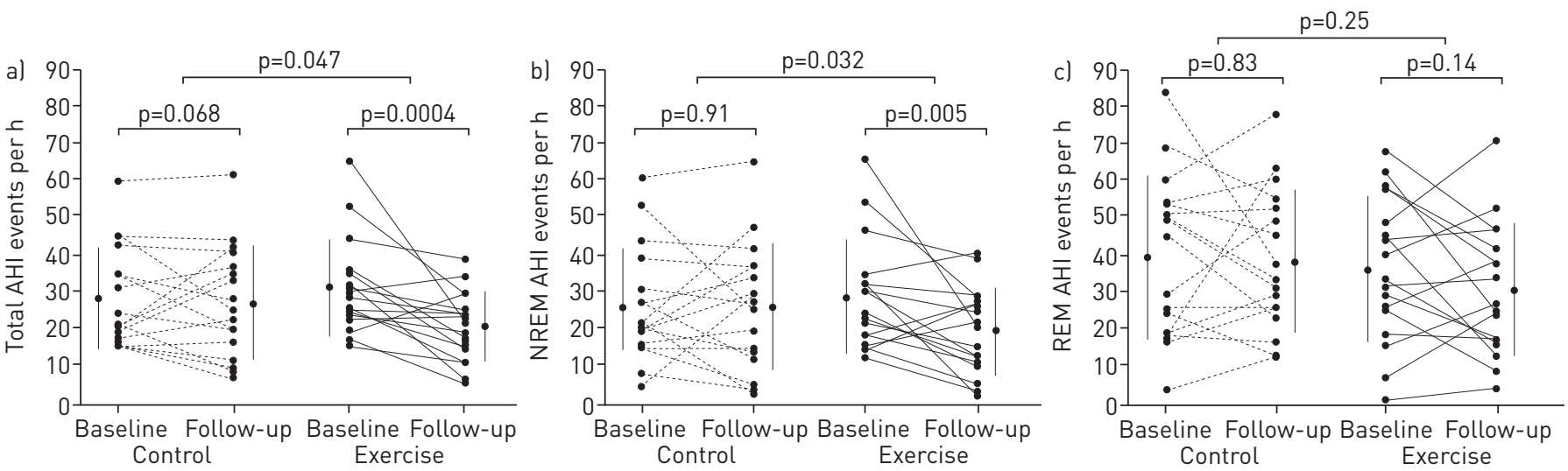

FIGURE 2 Change in al total apnoea-hypopnoea index (AHI), b) non-rapid eye movement (NREM) AHI and c) rapid eye movement (REM) AHI. 
TABLE 3 Polysomnographic data

\begin{tabular}{|c|c|c|c|c|c|}
\hline & \multicolumn{2}{|c|}{ Exercise $^{\#}$} & \multicolumn{2}{|c|}{ Control $^{\#}$} & Between-group p-value \\
\hline TST min & $282.2 \pm 61.3$ & $284.1 \pm 87.1$ & $325.6 \pm 43.9$ & $336.7 \pm 50.9$ & 0.63 \\
\hline N1 $\%$ & $17.1 \pm 15.7$ & $10.4 \pm 6.4$ & $11.1 \pm 3.8$ & $7.9 \pm 4.2 *$ & 0.48 \\
\hline N2 \% & $59.6 \pm 11.7$ & $58.4 \pm 18.7$ & $64.9 \pm 8.1$ & $63.4 \pm 9.5$ & 0.82 \\
\hline N3 \% & $9.5 \pm 9.3$ & $8.8 \pm 9.3$ & $9.4 \pm 6.5$ & $11.6 \pm 8.0$ & 0.41 \\
\hline Obstructive $\mathrm{AHI}$ events per $\mathrm{h}$ & $24.8 \pm 12.6$ & $17.2 \pm 9.4 *$ & $21.6 \pm 12.2$ & $21.8 \pm 13.5$ & 0.04 \\
\hline Central AHI events per $h$ & $6.2 \pm 7.9$ & $3.6 \pm 3.8$ & $6.8 \pm 14.6$ & $5.6 \pm 13.5$ & 0.28 \\
\hline Supine AHI events per $h$ & $49.2 \pm 25.5$ & $36.7 \pm 25.3$ & $40.1 \pm 15.7$ & $37.3 \pm 19.6$ & 0.25 \\
\hline Nonsupine AHI events per $h$ & $17.8 \pm 17.3$ & $14.8 \pm 9.9$ & $19.0 \pm 17.5$ & $28.9 \pm 25.7$ & 0.14 \\
\hline Periodic leg movements events per $h$ & $30.1 \pm 24.4$ & $39.9 \pm 32.1$ & $17.1 \pm 27.0$ & $13.9 \pm 21.0$ & 0.08 \\
\hline Supine time $\%$ TST & $47.8 \pm 30.1$ & $37.8 \pm 29.6$ & $46.6 \pm 30.5$ & $44.9 \pm 31.0$ & 0.43 \\
\hline
\end{tabular}

Data are presented as mean \pm SD unless otherwise stated. TST: total sleep time; N: non-rapid eye movement; REM: rapid eye movement; $\mathrm{AHI}$ : apnoea-hypopnoea index; $\mathrm{SaO}_{2}$ : arterial oxygen saturation. ${ }^{\#}$ : $\mathrm{n}=17$. *: $\mathrm{p}<0.05$ between baseline and follow-up (within group).

involved patients with CAD. Third, the physical activity levels employed were less strenuous and in accordance with the American College of Sports Medicine guidelines for exercise rehabilitation [24], and are therefore more attainable for the average person than in the previous study (i.e. $150 \mathrm{~min}$ of moderate-intensity exercise per week versus 630 min per week in the previous study).

We were not able to determine with certainty whether the reduction in AHI due to exercise was an acute effect related solely to reduced overnight rostral fluid shift or a more chronic exercise training effect. In favour of the former is that the AHI fell in relation to a reduced amount of fluid shifting rostrally from the legs to the neck in association with dilation of the upper airway overnight, whereas we did not observe any improvements in indices of physical fitness in the follow-up cardiopulmonary exercise test. In addition, the observation by REDOLF et al. [17] that in otherwise healthy men, wearing compression stockings for only 1 day caused significant reductions in LFV, overnight rostral fluid shift and AHI of a magnitude similar to our findings, also favours a more acute fluid shift-related effect.

We found that exercise training caused a modest $34 \%$ reduction in the AHI that was comparable to the effects of other interventions targeting fluid retention, such as compression stockings [18]. The most likely explanation for this is that exercise did not induce a large enough reduction in LFV and overnight LFV shift to eliminate OSA or CSA.

Another possible reason why exercise and other interventions that target fluid retention and overnight fluid shifts have induced only modest decrements in the AHI is that, in many patients, OSA and CSA are multifactorial in aetiology. For example, in some patients, structural/anatomical features that constrict space for the soft tissues around the pharynx are crucial to the development of OSA [25]. In others, reduced pharyngeal dilator muscle activity during sleep plays a significant role in inducing upper airway obstruction [26]. We found that exercise induced a significant reduction in the non-REM AHI but not the REM AHI, which is consistent the results of two previous studies targeting fluid shift [19, 27]. However, KLINE et al. [8] found that 3 months of exercise training resulted in a reduction in both REM and non-REM AHI. Therefore, it is possible that the 4-week exercise intervention was insufficient in duration to allow a reduction in REM AHI. Furthermore, we cannot explain the absence of decline in REM AHI by less supine time during REM sleep, as there was no significant change in the time spent in REM sleep in the supine position at the end of the intervention. Nonetheless, these findings suggest that rostral fluid shift may be more important in the pathogenesis of OSA during non-REM than REM sleep. This is possibly because during REM sleep, the main cause of OSA is the reduction in upper-airway dilator muscle activity, such that fluid accumulation in the neck would have little if any additional effect on upper-airway collapsibility [28]. Conversely, since the degree of reduction in upper-airway dilator muscle activity is not as great in non-REM as in REM sleep, fluid accumulation in the neck may have a more important role in the pathogenesis of OSA during non-REM sleep. These findings, taken together, suggest the possibility that there are different phenotypes of OSA, such that it might be possible to target specific pathophysiological aspects of sleep apnoea to provide individualised treatment. In particular, our results imply that interventions targeting fluid retention are more likely to attenuate sleep apnoea in non-REM than in REM sleep. 
TABLE 4 Changes in fluid volumes and upper-airway cross-sectional area (UA-XSA)

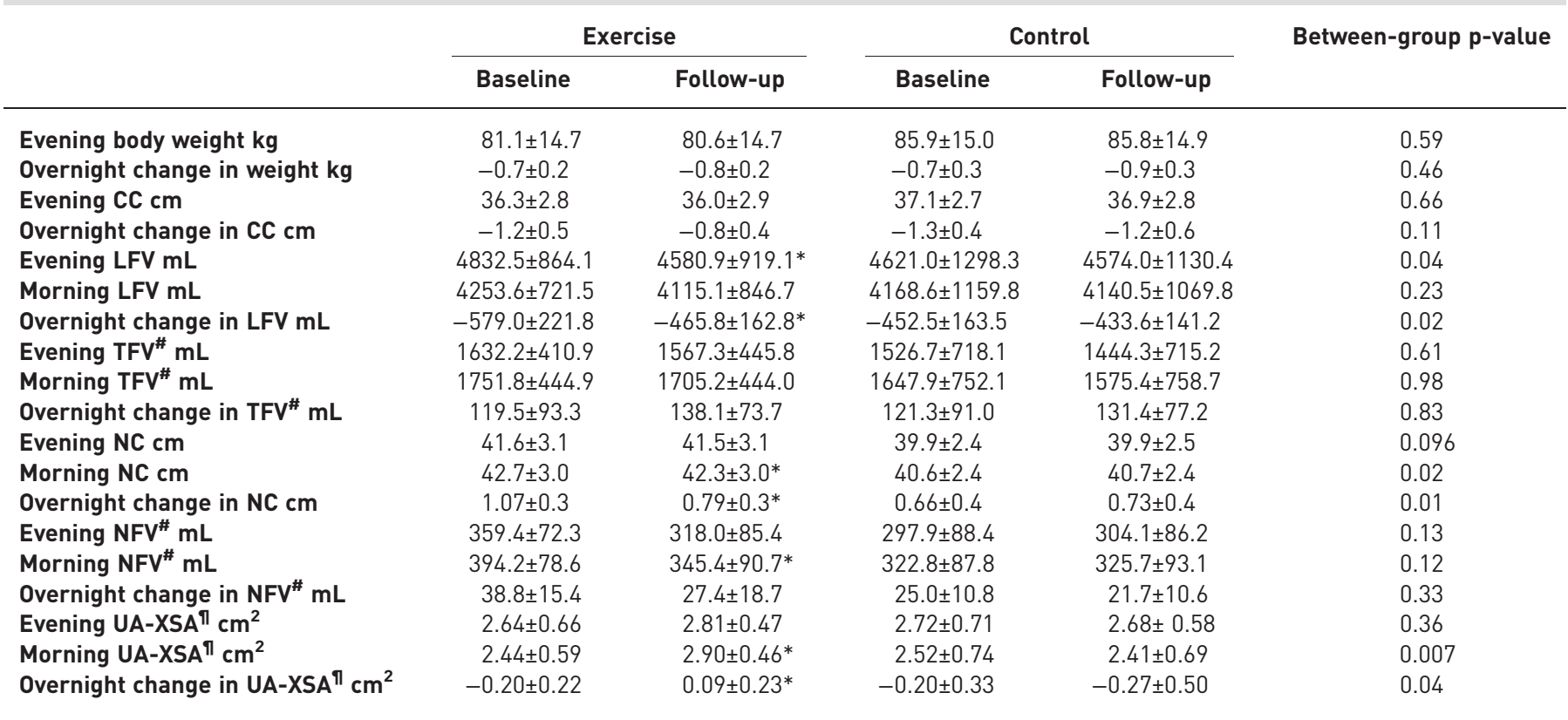

Data are presented as mean \pm SD unless otherwise stated. CC: calf circumference; LFV: leg fluid volume; TFV: thoracic fluid volume; NC: neck circumference; NFV: neck fluid volume. ${ }^{\#}$ : data for 14 and 13 participants in the exercise and control groups, respectively; ${ }^{\text {: }}$ : data for 11 and 12 participants in the exercise and control groups, respectively. *: $p<0.05$ between baseline and follow-up (within group).

\section{Limitations}

Our study had some limitations. First, the exercise intervention was relatively brief, such that a longer period may have caused a larger reduction in overnight rostral fluid shift and AHI. Second, the exercise intervention was limited to walking, so that it is possible that other forms of exercise such as cycling might have caused greater effects. Third, we did not have an objective measure of exercise in the control group during the 4 weeks, so that they may have increased their exercise. However, since they did not attend in-centre exercise training, they almost certainly exercised less than the intervention group. Finally, subjects were predominantly men who had OSA, so that results may not be generalisable to women and those with CSA.

\section{Conclusion}

In patients with $\mathrm{CAD}$ and moderate-to-severe sleep apnoea, exercise training reduced the AHI significantly, due to a fall in AHI during non-REM, but not REM, sleep. This occurred in association with a significant reduction in the amount of fluid shifting out of the legs overnight, and increases in UA-XSA overnight and in the morning, but in the absence of any improvement in physical fitness or weight loss. Taken together, these findings suggest that exercise training reduced the AHI by reducing the amount of fluid displaced from the legs into the neck overnight and a concomitant increase in upper-airway luminal size.

\section{References}

1 Alter DA, Oh PI, Chong A. Relationship between cardiac rehabilitation and survival after acute cardiac hospitalization within a universal health care system. Eur J Cardiovasc Prev Rehabil 2009; 16: 102-113.

2 Mora S, Cook N, Buring JE, et al. Physical activity and reduced risk of cardiovascular events: potential mediating mechanisms. Circulation 2007; 116: 2110-2118.

3 Peker Y, Hedner J, Kraiczi H, et al. Respiratory disturbance index: an independent predictor of mortality in coronary artery disease. Am J Respir Crit Care Med 2000; 162: 81-86.

4 Marin JM, Carrizo SJ, Vicente E, et al. Long-term cardiovascular outcomes in men with obstructive sleep apnoea-hypopnoea with or without treatment with continuous positive airway pressure: an observational study. Lancet 2005; 365: 1046-1053.

5 Wang H, Parker JD, Newton GE, et al. Influence of obstructive sleep apnea on mortality in patients with heart failure. J Am Coll Cardiol 2007; 49: 1625-1631.

6 Lanfranchi PA, Braghiroli A, Bosimini E, et al. Prognostic value of nocturnal Cheyne-Stokes respiration in chronic heart failure. Circulation 1999; 99: 1435-1440.

7 Kline CE, Crowley EP, Ewing GB, et al. The effect of exercise training on obstructive sleep apnea and sleep quality: a randomized controlled trial. Sleep 2011; 34: 1631-1640.

8 Ueno LM, Drager LF, Rodrigues AC, et al. Effects of exercise training in patients with chronic heart failure and sleep apnea. Sleep 2009; 32: 637-647. 
9 Yamamoto U, Mohri M, Shimada K, et al. Six-month aerobic exercise training ameliorates central sleep apnea in patients with chronic heart failure. J Card Fail 2007; 13: 825-829.

10 Bradley TD, Floras JS. Obstructive sleep apnoea and its cardiovascular consequences. Lancet 2009; 373: 82-93.

11 Yumino D, Bradley TD. Central sleep apnea and Cheyne-Stokes respiration. Proc Am Thorac Soc 2008; 5: 226-236.

12 Redolfi S, Yumino D, Ruttanaumpawan P, et al. Relationship between overnight rostral fluid shift and obstructive sleep apnea in nonobese men. Am J Respir Crit Care Med 2009; 179: 241-246.

13 Kasai T, Motwani SS, Yumino D, et al. Differing relationship of nocturnal fluid shifts to sleep apnea in men and women with heart failure. Circ Heart Fail 2012; 5: 467-474.

14 Yumino D, Redolfi S, Ruttanaumpawan P, et al. Nocturnal rostral fluid shift: a unifying concept for the pathogenesis of obstructive and central sleep apnea in men with heart failure. Circulation 2010; 121: 1598-1605.

15 Kasai T, Bradley TD, Friedman O, et al. Effect of intensified diuretic therapy on overnight rostral fluid shift and obstructive sleep apnoea in patients with uncontrolled hypertension. J Hypertens 2014; 32: 673-680.

16 Lyons OD, Chan CT, Yadollahi A, et al. Effect of ultrafiltration on sleep apnea and sleep structure in patients with end-stage renal disease. Am J Respir Crit Care Med 2015; 191: 1287-1294.

17 Redolfi S, Bettinzoli M, Venturoli N, et al. Attenuation of obstructive sleep apnea and overnight rostral fluid shift by physical activity. Am J Respir Crit Care Med 2015; 191: 856-858.

18 White LH, Lyons OD, Yadollahi A, et al. Effect of below-the-knee compression stockings on severity of obstructive sleep apnea. Sleep Med 2015; 16: 258-264.

19 Berry RB, Budhiraja R, Gottlieb DJ, et al. Rules for scoring respiratory events in sleep: update of the 2007 AASM Manual for the Scoring of Sleep and Associated Events. Deliberations of the Sleep Apnea Definitions Task Force of the American Academy of Sleep Medicine. J Clin Sleep Med 2012; 8: 597-619.

20 Rechtschaffen A, Kales AA. A Manual of Standardized Terminology, Techniques and Scoring System for Sleep Stages of Human Subjects. Bethesda, National Institutes of Health, 1968.

21 Kyle UG, Bosaeus I, De Lorenzo AD, et al. Bioelectrical impedance analysis-part II: utilization in clinical practice. Clin Nutr 2004; 23: 1430-1453.

22 Fredberg JJ, Wohl ME, Glass GM, et al. Airway area by acoustic reflections measured at the mouth. J Appl Physiol Respir Environ Exerc Physiol 1980; 48: 749-758.

23 Iftikhar IH, Kline CE, Youngstedt SD. Effects of exercise training on sleep apnea: a meta-analysis. Lung 2014; 192: $175-184$.

24 Thompson W, ed. American College of Sports Medicine Guidelines for Exercise Testing and Prescription. 8th Edn. Philadelphia, Wolter Kluwer, 2010.

25 Ryan CM, Bradley TD. Pathogenesis of obstructive sleep apnea. J Appl Physiol (1985) 2005; 99: 2440-2450.

26 Mezzanotte WS, Tangel DJ, White DP. Influence of sleep onset on upper-airway muscle activity in apnea patients versus normal controls. Am J Respir Crit Care Med 1996; 153: 1880-1887.

27 Yadollahi A, Gabriel JM, White LH, et al. A randomized, double crossover study to investigate the influence of saline infusion on sleep apnea severity in men. Sleep 2014; 37: 1699-1705.

28 Sauerland EK, Harper RM. The human tongue during sleep: electromyographic activity of the genioglossus muscle. Exp Neurol 1976; 51: 160-170. 